\title{
Depressão Pós-Parto Materna: Crenças, Práticas de Cuidado e Estimulação de Bebês no Primeiro Ano de Vida
}

\author{
Bárbara Camila de Campos \\ Olga Maria Piazentin Rolim Rodrigues \\ Universidade Estadual Júlio de Mesquita Filho - UNESP \\ São Paulo, SP, Brasil
}

\begin{abstract}
RESUMO
Após o nascimento a interação do recém-nascido com o mundo é facilitada pela mãe. Estudos indicam que mães deprimidas podem interagir pouco com seu bebê, gerando déficits comportamentais e cognitivos ao longo do desenvolvimento. Desta forma este estudo pretendeu descrever e relacionar o índice de depressão pós-parto apresentado por mães de bebês e as práticas e crenças sobre cuidado primário e estimulação. Foi utilizada a "Escala de Edinburgh de Depressão Pós-Parto - EPDS" e a "Escala de crenças parentais e práticas de cuidado (E-CPPC) na primeira infância". Os resultados obtidos com 132 mães indicaram sintomas de depressão para $29,5 \%$ da amostra. Com relação às práticas houve diferenças significativas entre os grupos clínicos e não clínicos na dimensão Estimulação, indicando que mães deprimidas podem interagir e estimular menos seus bebês. Desta forma têm-se aí um grupo de risco em que a díade deve ser cuidada garantindo saúde e um desenvolvimento adequado.
\end{abstract}

Palavras-chave: Depressão pós-parto; práticas e crenças maternas; cuidados primários; estimulação.

\section{ABSTRACT}

\section{Postpartum Depression Beliefs and Practices of Mother Care}

After birth the interaction between parents and children starts, studies indicate that depressed mothers can interact less with your baby, it can causing behavioral and cognitive deficits throughout development. This study aimed to describe and relate the postpartum depression index presented by mothers of babies and, practices and beliefs about primary care and stimulation. As a method was used the "Edinburgh Postpartum Depression Scale - EPDS" and the "Scale of parental beliefs and care practices (E-SCLC) in early childhood". The results indicated that 132 mothers had depression symptoms, $29.5 \%$ of the sample. At the practices were significant differences between clinical and non-clinical groups in Stimulation dimension, indicating that depressed mothers can interact and stimulate less their babies. Setting up a risk group in which the dyad should receive special care in order to ensure proper development.

Keywords: Postpartum depression; practices and maternal beliefs; primary care; stimulation.

\section{RESUMÉN}

\section{Depresión Posparto, Creencias y Prácticas de Cuidado de la Madre}

Después del nacimient do bebé la interacción se inicia, los estudios indican que las madres deprimidas pueden interactuar menos, esto puede causar déficits conductuales y cognitivos en desarrollo . Este estudio tuvo como objetivo describir y relacionar el índice de depresión posparto de madres, su prácticas y creencias acerca de la atención y la estimulación. Se ha utilizado como método la "Escala de Depresión Postparto Edimburgo - EPDS" y "Escala de creencias de los padres y las prácticas de atención (E-SCLC) en la primera infancia". Los resultados indicaron que 132 madres tenían síntomas de depresión $(29,5 \%)$. En las prácticas se encontraron diferencias entre los grupos clínicos y no clínico en dimensión de Estimulación, lo que indica que las madres deprimidas pueden interactuar y estimular menos a sus bebés. Son un grupo de riesgo, la díada debe recibir atención especial con el fin de garantizar el desarrollo adecuado.

Palabras-clave: Depresión posparto; prácticas y creencias maternas; cuidado primario; estímulo. 
A Organização Mundial da Saúde (WHO, 2012) indica que o índice de pessoas com sintomas relativos à depressão é de mais de 350 milhões em todo o mundo tratando-se de um fenômeno multicultural que vem afetando, anualmente, em torno de $5 \%$ da população mundial, sendo o Brasil o primeiro lugar no ranking da prevalência da doença em países em desenvolvimento (WHO, 2012). A depressão como doença é classificada, segundo o Manual Diagnóstico de Transtornos Mentais (DSM-V, 2013), como um Transtorno do Humor. No manual da Classificação Internacional das Doenças (CID-10, 1993) é classificada quanto à intensidade e predomínio de tipos de sintomas com a finalidade de quantificar a gravidade da doença e o nível de comprometimento do sujeito acometido.

Em relação à epidemiologia e a etiologia estudos indicam maior prevalência de depressão na população feminina; com relação a faixa etária há maior ocorrência após os 24 anos, em média, independente do gênero; aspectos sociais indicam que menor renda, desemprego, baixo nível de escolaridade e separação conjugal também são fatores de risco. (Holmes, 2001)

Dados sobre saúde mental e gênero indicam que não há diferenças significativas entre o risco para o desenvolvimento de doenças psiquiátricas entre homens e mulheres, porém, identifica-se variações em relação a predominância dos transtornos. Fatores relacionados à maior prevalência da depressão na população feminina são: psicossociais (historicamente ligados à maior vulnerabilidade social e dependência financeira; multiplicidade de papeis; problemas conjugais e familiares); história de distúrbios emocionais, como stress e ansiedade, pré-existentes; alterações hormonais (relação entre a diminuição do estrogênio e o desenvolvimento de depressão, característicos das fases pré-menstrual e pós-parto); Genética e a Resiliência. A depressão é considerada uma das doenças que mais gera gastos na saúde da mulher e afastamentos do trabalho (Figueira, Diniz, \& SilvaFilho, 2011).

Dentre as expressões dos transtornos depressivos nessa população encontram-se dados sobre o período da gravidez e a incidência e recorrência de depressão. Por ocasião do nascimento de um bebê, o período pós-parto é um evento estressor em potencial com comorbidade altamente associada à depressão. As alterações de humor características do pós-parto têm um espectro amplo relacionado à gravidade e comprometimento do indivíduo, sendo eles: o baby blues ou tristeza materna, a depressão pós-parto e a psicose pós-parto. Para o diagnóstico difencial têm-se, o baby blues ou tristeza materna como um quadro transitório que não configurase como transtorno e acomete em torno de $50 \%$ a $85 \%$ das mães até dez dias após o parto. É caracterizado por um quadro melancólico, com disforia, choro frequente, ansiedade, irritabilidade e dependência. Há hipóteses de que esse quadro seja desencadeado devido à intensa mudança dos níveis hormonais característicos do pósparto, somada ao stress do parto e às novas obrigações da maternidade. (Sit \& Wisner, 2009; Cantilino et al., 2010; Prenoveau et al., 2013)

A psicose pós-parto é um quadro mais grave e pouco frequente na população, acometendo uma em cada 1.000 mães. Na maioria das vezes tem comorbidade com o transtorno bipolar. Ocorre nas primeiras duas semanas após o nascimento do bebê, os sintomas envolvem ansiedade severa, alucinações e delírios; exigem acompanhamento terapêutico e medicamentoso intensivo além de supervisão por conta da possibilidade de suicídio ou agressões ao bebê. (Schardosim \& Heldt, 2011; DSM-V, 2013).

Finalmente, sobre a depressão com início no pósparto, transtorno depressivo no pós-parto ou depressão pós-parto materna (DPP-M), de acordo com o DSM-V (2013), ocorre nas primeiras quatro semanas após o parto, tendo, frequentemente, início durante a gestação. Com algumas especificidades o transtorno tem alta probabilidade de comorbidade com stress, ansiedade e sintomas obsessivo-compulsivos, há menor incidência de suicídio e a resposta a terapia farmacológica é lenta e recomenda-se a combinação de mais de um medicamento (Sit \& Wisner, 2009; Prenoveau et al., 2013).

Segundo a Organização Mundial da Saúde (WHO, 2009) a depressão pós-parto afeta $10 \%$ a $15 \%$ das mulheres em países desenvolvidos, além disso nas mulheres com episódio de depressão maior anterior tem o risco aumentado em $25 \%$ a $50 \%$. Uma análise dos estudos sobre a depressão pós-parto (DPP) mostra que há pouco consenso em relação aos dados sobre sua incidência (Santos, Silveira, \& Gualda, 2009; Cantilino et al., 2010; Figueira et al., 2011). No Brasil, podemos encontrar algumas variações, Cantilino et al. (2010), em um artigo de revisão encontrou dez estudos da última década que estimaram a prevalência da DPP no Brasil, as taxas encontradas nos estudos variaram de 7,2\% em Recife/PE a 43\% no Rio de Janeiro/RJ e uma pesquisa mais recente feita em São Paulo/SP obteve uma incidência de 30,3\% (Morais, Lucci, \& Otta, 2013). É importante ressaltar que os índices devem ser considerados levando em conta os dados culturais, étnicos e socioeconômicos de uma população, além dos fatores de risco e proteção existentes e não só grau de desenvolvimento de um país.

Pode-se destacar, também, que não há um consenso sobre o diagnóstico da DPP e o período em que ela ocorre. De acordo com o DSM-V, dá-se nas primeiras 
quatro semanas após o nascimento do bebê. Segundo o CID-10 aparece nas seis primeiras semanas, não havendo um período limite para a sua investigação. No que tange à metodologia, diferentes instrumentos são utilizados para rastreamento e diagnóstico da depressão pós-parto. Schardosim e Heldt (2011), em um artigo de revisão, encontraram estudos que utilizaram quatro tipos escalas para a triagem da DPP: a Edimburg Depression Postpartum Scale (EDPS); a Pospartum Depression Screening Scale (PDSS); a Hopkins Symptom Check List (SCL-25) e o SelfReport Questinnaire 20 (SRQ-20). Em um estudo posterior, Carvalho e Lima Salum (2014) identificaram 33 estudos nacionais e internacionais que utilizaram a EDPS, na última década, como instrumento de avaliação para o diagnóstico dos comportamentos característicos da depressão pós-parto.

A adaptação e validação da versão brasileira da EPDS feita por Santos, (1995); a escala é um instrumento de auto-registro, composto por dez itens pontuados em escala likert (zero a três) que variam de acordo com a presença ou a intensidade do sintoma e a mãe assinala as respostas que melhor descrevem o modo como tem se sentido na última semana. Seus itens incluem a investigações dos sintomas já listados como diagnósticos nos manuais de saúde mental. A somatória total dos escores resulta em 30 , sendo considerado de sintomatologia depressiva, neste estudo de validação, valor igual ou superior a 12. Estudos posteriores para a adaptação trans-cultural indicam pontos de corte diferentes e reduzidos para a identificação da DPP-M; Santos, Martins e Pasquali, (1999) num estudo com mães do centro-oeste brasileiro identificou o ponto de corte 11/12. No sul do país houve uma distinção no ponto de corte considerando a necessidade da escala, recomenda-se ponto de corte entre nove e 10 pontos como critério para o rastreio de DPP-M e entre 10 e 11 para a identificação de casos de DPP-M moderada ou grave (Santos et al., 2007).

Com relação à etiologia, estudos sugerem que os fatores de risco associados à DPP envolvem aspectos biológicos e psicossociais e afirmam que há semelhança entre os fatores de risco para a DPP na maioria das culturas e países; sendo os principais: episódio depressivo prévio, antes ou durante a gestação; histórico familiar de transtornos; conflitos familiares; exposição à eventos estressores durante a vida; pouco suporte social e financeiro; conflitos conjugais; gravidez na adolescência; gravidez indesejada; complicações no parto e dificuldades em lidar com o bebê decorrentes do comportamento, temperamento ou doenças. Enquanto fatores de proteção o suporte social adequado tanto da família, na relação conjugal ou de serviços que auxiliem na preparação física e psicológica para as mudanças advindas com a maternidade são variáveis relevantes. (Fonseca, Silva, \& Otta, 2010; Guedes et al., 2011; Murray, Halligan, \& Cooper, 2011; LeahyWarren, McCarthy, \& Corcoran, 2012).

A ocasião de nascimento do bebê, embora culturalmente associado a sentimentos positivos, é um período em que a mulher passa por muitos momentos de stress, constructo correlato à depressão. (Prenoveau et al., 2013). Desta forma, além da dificuldade e incapacitações características do transtorno, alterações de humor expressas no período após a gravidez podem colaborar para o surgimento de relações de contingencias aversivas; decorrentes das crenças sobre o que é adequado socialmente para o papel de mãe acompanhado pelo estigma dos transtornos mentais. A comunidade verbal tende a esperar que o nascimento do bebê seja um evento que naturalmente direciona a mulher para a maternagem (comportamentos com a função de garantir alimento, afeto e tranquilidade ao bebê) desta forma intensificam-se as dificuldades para aceitar comportamentos relacionados à tristeza ou depressão nesse período (Guerra, Braga, Quelhas, \& Silva, 2014).

Tornar-se mãe e a maternagem são processos comportamentais que envolvem diferentes momentos e experiências do indivíduo: história e relação com modelos parentais, padrões culturais, apoio social e o acesso às informações sobre desenvolvimento infantil, por exemplo. Desta forma, não é simplesmente o parentesco biológico que determina o comportamento materno tido como ideal, entretanto mães que não correspondem às expectativas sociais podem desenvolver um padrão comportamental característico de esquiva com o objetivo de esconder ou controlar respostas que reflitam esses sentimentos publicamente, o que pode ocasionar a manutenção de outros comportamentos encobertos típicos da depressão como a apatia, pouca sensibilidade as resposta do ambiente, culpa ou pensamentos recorrentes sobre algum problema (Evans, Heron, Lewis, Araya, \& Wolke, 2005; Gradvohl, Osis, \& Makuch, 2014).

Para Bowlby (2002), logo após o nascimento, quando o bebê inicia o contato com o ambiente, os sistemas comportamentais estão prontos para serem estimulados. Após o nascimento, as interações mãebebê ocorrem de maneira tênue; durante a interação, ao tocar, olhar, falar com o bebê geram estimulações recíprocas que promovem o ajustamento da díade ou relações de apego. Segundo Bowlby (1984) o apego é uma característica filogenética da interação da díade, é dividido em seguro, inseguro e evitante com base nas características e qualidade da responsividade materna que ocasionam a regulação emocional do bebê. Morais 
et al. (2013) e Gradvohl, Osis e Makuch (2014), ressaltam que a mãe ou o cuidador devem estabelecer papel de mediador na interação do recém-nascido com o mundo, apontando que, neste caso, a boa saúde da mãe é importante para que essa interação se dê de uma maneira positiva, possibilitando um desenvolvimento relacional, afetivo, motor e cognitivo adequado.

Martins, Vieira, Seidl-de-Moura e Macarini (2011) utilizaram a Escala de Crenças Parentais e Práticas de Cuidado (CPPC) para avaliar como uma amostra de mães brasileiras cuidava de seus filhos, levando em conta fatores sociodemográficos. A pesquisa indicou que as diferenças resultantes nas práticas de cuidados primários e estimulação estão ligadas aos diferentes modelos culturais e à disponibilidade materna.

Macarini, Martins, Minetto e Vieira (2010), em uma revisão sistemática sobre práticas parentais sugerem que há poucos estudos sobre essas práticas no primeiro ano de vida da criança, sendo que as etapas iniciais após o nascimento são fundamentais para o estabelecimento de intenções positivas ao longo de todo desenvolvimento. A presença da DPP pode impactar muitos sistemas, a qualidade de vida, a dinâmica familiar e a interação mãe-bebê, os recémnascidos são altamente vulneráveis e a depressão materna significa um risco para o desenvolvimento, pois pode comprometer a qualidade dos cuidados e da responsividade emocional da mãe.

Nos meses iniciais após o nascimento, as interações mãe-bebê ocorrem de maneira tênue e são necessárias para que o bebê estabeleça relações de apego seguro e mecanismos de regulação emocional. Alvarenga e Palma (2013) ao avaliar essa interação observam que mães deprimidas, além de apresentar altos níveis de stress, mantém interações intrusivas com seus filhos e tendem a apresentar mais práticas parentais negativas. Considerando este ambiente e os efeitos da condição na qualidade na interação estes fatores são considerados preditores para problemas no desenvolvimento, tornando essas crianças um grupo de risco importante (Picinini, Frizzo, Brys, \& Lopes, 2014).

Os estudos que examinam as primeiras trocas ou interações entre mães e bebês são, em geral, observacionais. Quanto à interação mãe-bebê, estudos indicam que mães com DPP interagem menos com seu bebê de forma adaptativa, são menos responsivas, estabelecem menos contato físico, usam de práticas educativas intrusivas e tendem a demonstrar menos sentimentos positivos e podem interromper a amamentação precocemente, quando comparadas com mães não deprimidas. Ao analisar o comportamento desses bebês as seguintes característica para o grupo clínico: exibem menos afeto positivo; mais afeto negativo; são menos ativos; têm menos vocalizações; desviam o olhar; apresentam mais comportamentos negativos (irritação, protestos, choro, expressões de tristeza e raiva); têm mais problemas de alimentação; alterações no sono e menor desenvolvimento motor. Avaliações longitudinais com mães deprimidas, procuraram identificar a influência do transtorno no pós-parto na qualidade da saúde mental e problemas de comportamentos pré-escolares de crianças. Os resultados indicaram que essas crianças, expostas a eventos adversos e instabilidade afetiva, são menos resilientes e mais propensas a apresentarem depressão, problemas de comportamento, atraso no desenvolvimento cognitivo. (Halligan, Murray, Martins, \& Cooper, 2007; Murray et al., 2010; Murray, 2011; Flores, Ramos, Moraes, \& Beltrami, 2012; Alvarenga, \& Palma, 2013; Ribeiro, Perosa, \& Padovani, 2014).

Os princípios do Sistema Único de Saúde, pela lei 8.080/90, estipulam que o novo modelo de assistência em saúde e saúde mental atenda a população também na prevenção, entendendo o processo de saúde-doença como algo social (Liberato, 2009). A emergência da depressão como um problema de saúde pública indica a importância da obtenção de dados precisos e rigor teórico em estudos que favoreçam o conhecimento a respeito do transtorno para que sejam traçadas estratégias em relação a identificação e diagnóstico precoce além da possibilidade de prevenção diante da presença dos fatores de risco, como da depressão pósparto, visando diminuir os prejuízos sobre a mãe e os efeitos negativos em longo prazo para a díade.

\section{OBJETIVO}

O presente estudo teve como objetivo identificar o índice de depressão pós-parto materna de bebês entre dois e quatro meses de idade, descrever as práticas de cuidado primário e estimulação utilizadas por elas assim como suas crenças à respeito da importância destas e, por fim, relacionar a presença ou ausência de comportamentos indicativos para depressão com a frequência de realização das práticas e a importância relatada.

Sendo assim, a importância da avaliação precoce da depressão pós-parto e do relato das mães a respeito de suas práticas pode oferecer pistas para intervenções com essa população. Intervenções pontuais, incluindo o encaminhamento para serviços especializados, assim que os sintomas forem identificados, podem resultar em melhora da responsividade materna ao seu bebê. O delineamento de estratégias que abarquem os fatores de proteção e possibilitam que as crianças tenham um desenvolvimento adequado e sofram menos prejuízos garantindo a qualidade das interações entre a díade. 


\section{MÉTODO}

\section{Sujeitos}

Participaram desta pesquisa 132 mães de bebês com dois a seis meses que frequentavam um projeto de extensão sobre o "Acompanhamento do desenvolvimento de bebês: avaliação e orientação aos pais", que funciona no Centro de Psicologia Aplicada, da UNESP de Bauru e por ocasião do convite aceitaram participar da pesquisa que parte do projeto "Variáveis maternas e do bebê: correlação entre interação e desenvolvimento infantil".

\section{Instrumentos}

Para a identificação da depressão pós-parto foi utilizada a "Escala de Edinburgh de Depressão PósParto" - Edinburgh Postpartum Depression Scale EPDS de (Cox, Holden, \& Sagovsky, 1987); validada para a população brasileira por Santos (1995). Optouse, neste estudo, utilizar, por ter melhores índices de predição, score 12 como ponto de corte para indicativo de DPP-M, diante deste critério as participantes foram separadas em grupos Clínico e Não clinico para diagnóstico de depressão.

Os cuidados com o bebê foram avaliados a partir da "Escala de crenças parentais e práticas de cuidado (E-CPPC) na primeira infância” (Suizzo, 2002, validada por Martins et al., 2010), para crianças de zero a seis anos de idade que engloba práticas de cuidado e estimulação utilizadas por pais, bem como crenças que os mesmos possuem e orientam seus comportamentos. $\mathrm{O}$ instrumento consiste em 36 itens, que descrevem comportamento maternos em duas subescalas: "Práticas Realizadas pela Mãe" (PR) e "Importância Atribuída às Práticas" (IAP), em uma escala likert. No primeiro conjunto, para cada assertiva, a mãe deve assinalar o quanto realiza ou a importância que atribui ao item, numa escala de cinco pontos $(1=$ "não concordo"; 5="é extremamente importante"). Entre as questões há dois diferentes domínios do cuidado parental: (a) Estimulação: relacionadas com práticas que estimulem o desenvolvimento cognitivo e motor da criança, como, por exemplo, oferecer brinquedos que estimulem seus sentidos e ler histórias para a criança e, (b) Cuidados: relacionada com práticas orientadas para boa higiene da criança em lugares públicos e ênfase nos comportamentos socialmente aceitáveis, como manter a criança limpa e adequadamente arrumada.

\section{Procedimento}

A pesquisa foi aprovada pelo pelo Comitê de Ética da Faculdade de Ciências da Universidade Estadual
Paulista - UNESP (Processo no $11187 / 46 / 01 / 2012$ ) e as participantes assinaram um Termo de Consentimento Livre e Esclarecido. Em seguida do aceite, as mães eram convidadas a preencherem os instrumentos numa sala reservada e após a finalização da aplicação elas participavam do atendimento do Projeto de Extensão para avaliavar o desenvolvimento de seus bebês.

\section{Análise dos dados}

Os dados sociodemográficos foram analisados de maneira descritiva. Para realizar os testes estatísticos dos dados obtidos nas escalas foi utilizado o SPSS (Statistical Package for Social Sciences 21.0) e, os dados foram analisados pelo teste T de Student, com o objetivo de verificar se há diferenças significativas (intervalo de confiança de $95 \% ; \mathrm{p}=0,05$ ) entre a frequência de realização dos comportamentos, tanto geral quanto em amostras pares do grupo Clínico e Não Clínico.

\section{RESULTADOS}

A idade média das mães entrevistadas foi de 27 anos (idade mínima: 15 anos; máxima: 40; $d p=6,26$ ), e a média de idade das crianças foi de três meses (Mediana=3; dp=0,70). Com relação ao sexo do bebê foram 73 bebês do sexo masculino e 59 do sexo feminino.

Do total das 132 mães que responderam a EDPS, $70,5 \%$ (n=93) das mães compuseram o grupo Não Clínico (G1), ou seja, tiveram pontuação abaixo de 11 pontos. O restante, $29,5 \%(\mathrm{n}=39)$ manifestou sintomas de depressão pós-parto e faz parte do grupo Clínico (G2), apresentando pontuação maior ou igual a 12.

As análises da "Escala de Crenças Parentais e Práticas de Cuidado (E-CPPC) na primeira infância", observa-se na Tabela 1 que quanto aos Cuidados Primários, realizados ou a importância que atribuem a eles, todas as participantes relataram que os realizam com frequência alta $(78,03 \%)$ e, também, acham importante realizá-los (82,54\%). A maioria das mães está na média ou acima quando relatam sobre o quanto realizam as práticas de Cuidados Primários e quanto à importância que dão a eles.

Os dados referentes às crenças e práticas sobre Estimulação, para a amostra apontam que 63,63\% relataram que estimulam seus filhos com alta frequência e 90,90\% das mães declararam achar a estimulação importante. Comparando as práticas realizadas e a importância dada à elas, tanto na dimensão de cuidados quanto a de estimulação, a amostra toda teve índices maiores quando relataram sobre a importância atribuída à prática. 
TABELA 1

Crenças e práticas sobre os Cuidados Primários e Estimulação (n=132)

\begin{tabular}{lcccc}
\hline \multirow{2}{*}{ Classificação } & \multicolumn{2}{c}{$\begin{array}{c}\text { "Práticas Realizadas } \\
\text { pela Mãe" }\end{array}$} & $\begin{array}{c}\text { "Importância Atribuida } \\
\text { às Práticas" }\end{array}$ & $($ IAP) \\
\cline { 2 - 5 } & $n$ & $\%$ & $n$ & $\%$ \\
\hline Cuidados & & & & \\
32-34 pontos: abaixo da média & 8 & 6,06 & 2 & 1,51 \\
35-37 pontos: na média & 21 & 15,90 & 21 & 15,90 \\
38-40 pontos: acima da média & 103 & 78,03 & 109 & 82,54 \\
Estimulação & & & & \\
20-29 pontos: abaixo da média & 12 & 9,09 & 1 & \\
30-39 pontos: na média & 36 & 27,27 & 11 & 0,75 \\
40-50 pontos: acima da média & 84 & 63,63 & 120 & 8,33 \\
\hline
\end{tabular}

A Tabela 2 apresenta a comparação entre as médias obtidas pelo grupo geral em cada uma das dimensões, Cuidados e Estimulação e entre Práticas realizadas e a Importância atribuída à elas. Observa-se que as mães realizam e consideram importante as práticas de cuidado primário em frequência semelhante. Porém, na dimensão Estimulação houve diferença estatisticamente significante $(p=0,000)$ nas médias do grupo, indicando que as mães estimulam seus bebês numa frequência menor do que consideram importante realizar essa prática. Analisou-se, também, as diferenças observadas entre Práticas realizadas e a Importância dada entre Cuidados e Estimulação. No primeiro caso os dados apontaram que as médias na realização das práticas não diferem independente da dimensão. Porém quando observamos as médias relacionadas às crenças há significância $(\mathrm{p}=0,000)$, indicando que a importância dada aos comportamentos de Cuidados Primários é menor do que as Práticas de Estimulação.

Na Tabela 3, considerou-se a divisão da amostra por grupos não clínicos (G1) e clínicos (G2) a partir dos resultados obtidos na EPDS comparando a média de desempenho de cada um deles na E-CPPC. Quanto às práticas realizadas, na dimensão Cuidados Primários não se observou diferença significativa entre os grupos, porém houve uma tendência $(p=0,094)$ com média maior para o G1. Na dimensão Estimulação houve diferença estatisticamente significativa $(p=0,000)$ com média maior para o $\mathrm{G} 1$, um dado relevante indicando que as mães do G1 estimulam com mais frequência seus bebês. Quanto à importância atribuída a não houve diferença entre os grupos apenas uma tendência $(p=0,057)$ entre as mães do G2 (Clínico) quanto as Práticas de Cuidado. $\mathrm{Na}$ análise intragrupos das dimensões, houve diferença significativa $(\mathrm{p}=0,000 \mathrm{e}$ $\mathrm{p}=0,008)$ em todas as médias para a relevância dada à dimensão Estimulação.

A análise entre os Grupos Clínico e Não Clínico, foi realizada a partir da frequência relativa dos participantes, de acordo com a classificação proposta para este estudo obtida na E-CPPC, para a dimensão Estimulação, que apresentaram diferenças significativas entre os grupos nas análises estatísticas conduzidas (Tabela 4). No grupo de Práticas abaixo da média e na média $(20-29$ e de 30 a 39 pontos) as mães que fazem parte do Grupo Clínico compõem $64,10 \%$ desse grupo. Percebe-se que o número de mães com práticas de Estimulação mais altas (de 40 a 50 pontos) é, em sua maioria (75,26\%), do G1, ou seja, mães que não tiveram o diagnóstico de depressão pós-parto na EPDS.

TABELA 2

Comparação entre as médias e desvio padrão entre crenças e práticas sobre os Cuidados Primários e Estimulação

\begin{tabular}{lccc}
\hline \multicolumn{1}{c}{ Dimensões } & $\begin{array}{c}\text { "Práticas Realizadas } \\
\text { pela Mãe" }(\text { PRM })\end{array}$ & $\begin{array}{c}\text { "Importância Atribuída } \\
\text { às práticas" (IAP) }\end{array}$ & $P$ \\
\hline Cuidados Primários & $38,95(1,73)$ & $39,17(5,57)$ & 0,205 \\
Estimulação & $38,40(7,37)$ & $45,82(4,17)$ & 0,000 \\
$P$ & 0,575 & 0,000 & \\
\hline
\end{tabular}


TABELA 3

Resultados da EPDS e as práticas e crenças comparando os grupos Clínico e Não Clínico e comparando a média e o desvio padrão das Práticas Realizadas e a importância atribuída a ela, em Cuidados Primários e Estimulação

\begin{tabular}{lccc}
\hline \multicolumn{1}{c}{ Dimensões } & G1 (Não Clínico) & G2 (Clínico) & $P$ \\
\hline Práticas Realizadas & & & \\
Cuidados Primários & $38,59(0,76)$ & $38,15(2,69)$ & 0,094 \\
Estimulação & $42,41(4,99)$ & $34,79(6,24)$ & 0,000 \\
$p$ & 0,000 & 0,008 & \\
Importância Atribuida à Prática & & & \\
Cuidados Primários & $40,43(5,24)$ & $39,00(3,93)$ & 0,087 \\
Estimulação & $44,92(4,18)$ & $45,76(4,54)$ & 0,182 \\
$p$ & 0,000 & 0,000 & \\
\hline
\end{tabular}

TABELA 4

Distribuição das mães dos grupos Clínico e Não Clínico a partir da classificação das Práticas de Estimulação

\begin{tabular}{lccccccc}
\hline & \multicolumn{7}{c}{ Pontos na E-CPPC } \\
\hline & & $20-29$ & & $30-39$ & & $40-50$ \\
\hline & $n$ & $\%$ & $n$ & $\%$ & $n$ & $\%$ \\
\hline G1 (Não Clínico) & $\mathrm{n}=93$ & 4 & 4,31 & 19 & 20,43 & 70 & 75,26 \\
G2 (Clínico) & $\mathrm{n}=39$ & 8 & 20,52 & 17 & 43,58 & 14 & 35,90 \\
\hline
\end{tabular}

\section{DISCUSSÃO}

As alterações hormonais, variações no humor e a mudança na rotina podem explicar a tristeza ou dificuldade de lidar com sentimentos, característicos do momento pós-parto, porém quando essas mudanças, típicas do baby blues, permanecem e começam a interferir na rotina e na interação do bebê há a necessidade de cuidar da díade.

Da amostra de mães participantes deste estudo observou-se uma incidência de $29,5 \%$ ( $n=39)$ de mães que manifestaram sintomas de depressão pós-parto. Este dado se aproxima do que a Organização Mundial da Saúde prevê para países em desenvolvimento, um índice de $40 \%$ de mães com esse indicativo (WHO, 2009). Entretanto há de se ressaltar que é preciso critério ao considerar essa estimativa, observarva-se variações amplas nos dados de prevalência encontrados em diferentes estudos. Em um estudo de revisão brasileiro, o índice variou de 15 a 28\% (Lobato, Moraes, \& Reichenheim, 2011); posteriormente, uma pesquisa de Morais et al. (2013), encontrou 30,3\% das mães com depressão pós-parto.

Figueira et al. (2011) apontam que o papel da cultura e a as crenças sobre o período da maternidade podem exercer influência no comportamento da mulher em evitar buscar ajuda ou falar sobre sentimentos negativos. Os sintomas depressivos, em geral, caracterizam-se por alteração do humor, cansaço ou falta de energia para realizar atividades, dificuldade de concentração e mudança na sensação de prazer relacionadas às situações que eram consideradas agradáveis. Além disso, em alguns casos há alteração no sono, cansaço acentuado, diminuição da autoestima e sentimento de culpa (DSM-V, 2013). Entretanto, mesmo com os sintomas depressivos evidenciados pela ocasião da aplicação da escala e o convite para que as mães passassem por uma avaliação psicológica no próprio centro, poucas aderiram ou associavam à dificuldades na maternidade.

Schardosin e Heldt (2011) e Carvalho e Lima Salum (2014) apontam, ainda, que no Brasil não há instrumentalização adequada dos profissionais da saúde a respeito do transtorno e de como identificar a depressão no período do pós-parto. Desta forma, a utilização da EPDS, um instrumento validado em muitos países e amplamente utilizado e de fácil aplicação, é uma alternativa de instrumento para triagem, diagnóstico e encaminhamento a ser utilizada pelos profissionais da saúde.

Considerando o desempenho na E-CPPC, para a amostra toda, observou-se que, de acordo com a 
classificação proposta para este estudo, em Cuidados Primários, a maioria das mães relatou que fazem e, mais ainda, que acham importante fazê-lo, tais resultados comprovam os encontrados por dimensão (Martins et al., 2010; Martins et al., 2011). Os Cuidados Primários estão diretamente relacionados à uma dimensão filogenética e têm, como consequência, a redução do desconforto e a garantia da sobrevida do bebê. Ao comparar o total absoluto das médias na Tabela 2, as mães declararam atribuir importância a estes cuidados.

$\mathrm{Na}$ dimensão Estimulação mais de $90 \%$ das mães relataram achar que é importante fazê-lo, mas pouco mais de $60 \%$ realmente o fazem, dado que foi estatisticamente significante ao comparar as médias da dimensão na Tabela 2. Desta forma, há indícios de que, embora haja conhecimento das mães acerca de comportamentos importantes que favorecem o desenvolvimento, eles ainda não estão bem estabelecidos enquanto práticas maternas, como por exemplo: carregar no colo, ver livrinhos, conversar, pendurar brinquedos no berço.

Em relação a importância das duas dimensões avaliadas na escala, os autores indicam que não há valoração e o que deve ser considerado são suas funções específicas e influências no desenvolvimento global da criança. Keller (2007) ressalta que as crenças e práticas tanto de cuidado quanto de estimulação do bebê são aprendidas socialmente. Desta forma, a informação pode ser um meio de articulação entre a cultura e crenças em busca de uma modificação nas práticas dessas mães. Incentivar a elaborarem estratégias para passar momentos de interação com qualidade com seus bebês, como por exemplo, aproveitando os momentos de cuidado, para estimulá-los, incentivando comportamentos coadjuvantes para o desenvolvimento infantil.

Em Estimulação, observou-se diferenças significativas quando se referiu às práticas, a amplitude das médias entre os grupos $(\mathrm{G} 1=42,41 ; \mathrm{G} 2=34,79)$ é um dado importante sobre essa população, indicando que mães deprimidas estimulam significativamente menos seus bebês. Segundo Bowlby (2002) o papel de mediação e de estimulação da mãe é fundamental nos primeiros anos de vida levando em conta tanto fatores biológicos, cognitivos e afetivos, é nesse período que o vínculo e o apego com a mãe se forma. Além disso, Morais et al. (2013) ressaltam o papel da estimulação no desenvolvimento adequado do cérebro ampliando a possibilidade de redes e conexões neurais. Os dados obtidos neste estudo sobre as práticas de estimulação entre os grupos, confirmam os achados de outros que indicam que mães deprimidas têm uma tendência a interagirem menos com seus bebês o que pode ocasionar déficit nessas primeiras interações além de ser preditor para outros problemas de comportamento ao longo do desenvolvimento (Murray, 2010; Goodman \& Rouse, 2010; Lobato et al, 2011; Alvarenga \& Palma, 2013; Ribeiro et al., 2014; Picinini et al., 2014).

Com relação à importância atribuída aos comportamentos não houve diferença significativa nas dimensões e entre os grupos. Independente da presença/ ausência da depressão, em ambos os grupos, as mães atribuem mais importâncias às práticas de Estimulação.

Ao analisar mais detalhadamente pode-se traçar um perfil distinto dos grupos. A maioria das mães do G1 (75\%) têm pontuação acima da média e estimulam seus filhos com alta frequência. Já as mães do G2 têm a maior parte dos sujeitos distribuída entre a média $(43 \%)$ e acima da média $(35 \%)$, ou seja, a frequência com que realizam esses comportamentos é significativamente menor. Desta forma, o ambiente imediato do quadro depressivo pode influenciar na prática de comportamentos relacionados a estimulação. Mães, por ocasião da depressão, podem ter dificuldades em responder de maneira adequada e com qualidade, embora saibam da importância disso e a declarem enquanto crença (Macarini et al., 2010; Morais et al., 2013; Gradvohl et al., 2014; Ribeiro et al., 2014)

\section{CONSIDERACOES FINAIS}

O objetivo dessa pesquisa foi investigar a incidência da Depressão Pós-parto Materna e a influência desta nas práticas parentais e no desenvolvimento dos bebês. O número de mães que relataram comportamentos indicativos de depressão encontrado é elevado, porém, confirma os resultados de outros estudos.

As práticas relacionadas à realização dos cuidados primários foram elevadas e, no geral, positivas. Acredita-se que, como são comportamentos relativos às práticas de cuidados básicos relacionados à sobrevivência, saúde e higiene, esse fato pode estar relacionado com a possibilidade de as mães contarem com uma rede de apoio que as auxilie nesses cuidados, como por exemplo, familiares ou o companheiro. A relação observada entre as práticas de Estimulação e a presença de depressão é importante, uma vez que essa dimensão inclui comportamentos relacionados à facilitação de experiências diversas ao bebê, como de, por exemplo, explorar seu corpo e o ambiente. Sabe-se que o desenvolvimento infantil nos primeiros anos de vida depende intimamente do funcionamento da díade $\mathrm{e}$, as práticas relacionadas aos comportamentos de estimulação são importantes para o desenvolvimento adequado do bebê em todas as áreas. 
Tendo em vista que o ambiente de interação dos bebês é limitado pela disponibilidade materna ou do cuidador, a presença de uma mãe com comportamentos depressivos e pouco responsiva pode gerar déficits na interação da díade. A forma com que a criança interage com o mundo e a sua regulação emocional e autocontrole estão sujeitos à maneira de como essa relação é estabelecida. Diante de uma mãe pouco responsiva e sensível aos sinais do bebê e que estimula pouco, a possibilidade de haver algum prejuízo no desenvolvimento e na vinculação da díade é maior, têmse aí um grupo de risco e ambos devem ser cuidados.

\section{REFERÊNCIAS}

Alvarenga, P. \& Palma, E. M. S. (2013). Indicadores de Depressão Materna e a Interação Mãe-Criança aos 18 Meses de Vida. Psico, 44(3), 402-410.

Bowlby, J. (2002) Primórdios do comportamento do apego. In J. Bowlby. Apego e perda: apego (pp. 329-69). São Paulo: Martins Fontes.

Bowlby, J. (1984a). Apego. São Paulo: Martins Fontes.

Cantilino, A., Albuquerque, C. F., Paes, T. L. C., Montenegro, J. A., Peres, A. C., \& Sougey, E. B. (2010). Postpartum depression in Recife - Brazil: prevalence and association with bio-socio-demographic factors. Jornal Brasileiro de Psiquiatria, 59(1), 1-9. http://dx.doi.org/10.1590/S0047-20852010000100001

Cantilino, A., Albuquerque, C., \& Cantilino, G. (2003). Validação da escala de depressão pós-parto de Edinburgh (versão em português) no Nordeste do Brasil. Anais do XXI Congresso Brasileiro de Psiquiatria, Goiânia, GO.

Carvalho, F. A. \& Lima Salum, M. (2014). Relação entre Depressão Pós-Parto e Apoio Social: Revisão Sistemática da Literatura. Psico, 45(4), 463-474. http://dx.doi.org/10.15448/1980-8623.2014.4.15423

DSM-V: Diagnostic and Statistical Manual of Mental Disorder. (2013). American Psychiatric Association (APA).

Evans, J., Heron, J., Lewis, G., Araya, R., \& Wolke D. (2005). Negative self-schemas and the onset of depression in women: longitudinal study. The British Journal of Psychiatry, 186(4), 302-307. http://dx.doi.org/10.1192/bjp.186.4.302

Figueira, P. G., Diniz, L. M., \& Silva Filho, H. C. (2011). Características demográficas e psicossociais associadas à depressão pós-parto em uma amostra de Belo Horizonte. Revista de Psiquiatria do Rio Grande do Sul, 33(2), 71-75. http://dx.doi.org/10.1590/S0101-81082011005000009

Figueira, P. G., Diniz, L. M., \& Silva-Filho, H. C. (2011). Características demográficas e psicossociais associadas à depressão pós-parto em uma amostra de Belo Horizonte. Revista de Psiquiatria do Rio Grande do Sul (Porto Alegre), 33(2), 71-75. http://dx.doi.org/10.1590/S0101-81082011005000009

Flores, M. R., Ramos, A. P., Moraes, A., \& Beltrami, L. (2012). Associação entre indicadores de risco ao desenvolvimento infantil e estado emocional materno. Revista CEFAC, 15(02), 348-360. http://dx.doi.org/10.1590/S151618462012005000046

Fonseca, V. R. J. M., Silva, G. A., \& Otta E. (2010). Relação entre depressão pós-parto e disponibilidade emocional materna. Cadernos de Saúde Pública, 26 (4), 738-746. http://dx.doi.org/10.1590/s0102-311x2010000400016

Goodman, S. H. \& Rouse, M. H. (2010). Perinatal depression and children: A developmental perspective. Encyclopedia on early childhood development, Montreal, Quebec: Centre of Excellence for Early Childhood Development, 1-7.

Gradvohl, S. M. O., Osis, M. J. D., \& Makuch, M. Y. (2014). Maternidade e formas de maternagem desde a idade média à atualidade. Pensando famílias, 18 (1), 55-62.

Guedes, A. C. E., Kami, C. T., de Vargas Cavalli, L. K., Nicolaou, S. K., Hess, V. B., \& Maluf, E. M. C. P. (2011). Depressão pós-parto: incidência e fatores de risco associados. Revista de Medicina, 90(3), 149-154. http://dx.doi. org/10.11606/issn.1679-9836.v90i3p149-154

Guerra, M. J., Braga, M. C., Quelhas, I., \& Silva, R. (2014). Promoção da saúde mental na gravidez e no pós-parto. Revista Portuguesa de Enfermagem de Saúde Mental (SPE1), 117-124.

Halligan, S. L., Murray, L., Martins, C., \& Cooper, P. J. (2007). Maternal depression and psychiatric outcomes in adolescent offspring: a 13-year longitudinal study. Journal of affective disorders, 97(1), 145-154. http://dx.doi org/10.1016/j.jad.2006.06.010

Holmes, D. S. (2001). Psicologia dos transtornos mentais (3a ed.). Porto Alegre: Artmed.

Keller, H. (2007). Culture of Infancy. Mahwah, New Jersey; London: Lawrence Erlbaum Associates Publishers.

Leahy-Warren, P., Mccarthy, G., \& Corcoran, P., (2012) First-time mothers: social support, maternal parental selfefficacy and postnatal depression. Journal of Clinical Nursing, 13(3), 88-397. http://dx.doi.org/10.1111/j.13652702.2011.03701.x

Liberato, M. D. M. (2009). Desinstitucionalizar é ultrapassar fronteiras sanitárias: o desafio da intersetorialidade e do trabalho em rede. Cadernos Brasileiros de Saúde Mental, 2009.

Lobato, G., Moraes, C. L., \& Reichenheim, M. E. (2011). Magnitude da depressão pós-parto no Brasil: uma revisão sistemática. Revista Brasileira de Saúde Materno Infantil, 11(4), 369-379. http://dx.doi.org/10.1590/S151938292011000400003 
Lobato, Gustavo, Moraes, Claudia L, \& Reichenheim, Michael E. (2011). Magnitude da depressão pós-parto no Brasil: uma revisão sistemática. Revista Brasileira de Saúde Materno Infantil, 11(4), 369-379. http://dx.doi.org/10.1590/ S1519-38292011000400003

Macarini S. M., Martins, G. D. F., Minetto. M. F., M. F. J., \& Vieira, M. L. (2010). Práticas parentais: uma revisão da literatura brasileira. Arquivos Brasileiro de Psicologia, 62(1), 119-134.

Martins, G. D. F., Macarini, S. M., Vieira, M. L., Seidl-de-Moura, M. L., Bussab, V. L. R., \& Cruz, R. M. (2010). Construção e validação do Inventário de Crenças Parentais sobre Práticas de Cuidado (CPPC) na primeira infância. Psico-USF, 15, 23-34. http://dx.doi.org/10.1590/S1413-82712010000100004

Martins, G. D. F., Vieira, M. L., Seidl-de-Moura, M. L., \& Macarini, S. M. (2011). Crenças e práticas de cuidado entre mães residentes em capitais e pequenas cidades Brasileiras. Psicologia Reflexão e Critica, 24(9), 692-701.

Morais, M. L. S., Lucci, T. K., \& Otta, E.(2013). Postpartum depression and child development in first year of life. Estudos de Psicologia, 30(1) 7-17. http://dx.doi.org/10.1590/s0103-166x2013000100002

Murray, L., Arteche, A., Fearon, P., Halligan, S., Goodyer, I., \& Cooper, P. (2011). Maternal postnatal depression and the development of depression in offspring up to 16 years of age. Journal of the American Academy of Child \& Adolescent Psychiatry, 50(5), 460-470. http://dx.doi.org/10.1016/j.jaac.2011.02.001

Murray, L., Halligan, S., \& Cooper, P. (2010). Effects of postnatal depression on mother-infant interactions and child development. Wiley-Blackwell Handbook of Infant Development, 2(2), 192-220. http://dx.doi. org/10.1002/9781444327588.ch8

Piccinini, C. A. P., Frizzo, C. A., Brys, G. B. I., \& Lopes, R. C. S. (2014). Parenthood in the context of maternal depression at the end of the infant's first year of life. Estudos de Psicologia (Campinas), 31(2), 203-214. http://dx.doi. org/10.1590/0103-166X2014000200006

Prenoveau, J., Craske, M., Counsell, N., West, V., Davies, B., Cooper, P., \& Stein, A. (2013). Postpartum GAD is a risk factor for postpartum MDD: the course and longitudinal relationships of postpartum GAD and MDD. Depression and anxiety, 30(6), 506-514. http://dx.doi.org/10.1002/da.22040

Ribeiro, D. G., Perosa, G. B., \& Padovani, F. H. P. (2014). Saúde mental, interação mãe-criança e desenvolvimento ao final do primeiro ano de vida. Paidéia (Ribeirão Preto), 24(59), 331-339. http://dx.doi.org/10.1590/1982-43272459201407

Santos, I. S., Matijasevich, A., Tavares, B. F., Barros, A. J., Botelho, I.P., Lapolli, C., Magalhães, P. V., Barbosa, A. P., \& Barros, F. C., (2007). Validation of the Edinburgh Postnatal Depression Scale (EPDS) in a sample of mothers from the 2004 Pelotas Birth Cohort Study. Cad Saúde Pública, 23, 2577-2588. http://dx.doi.org/10.1590/S0102311X2007001100005

Schardosim, J. M. \& Heldt, E. (2011). Escalas de rastreamento para depressão pós-parto: uma revisão sistemática. Revista Gaúcha de Enfermagem (Porto Alegre), 32(01), 159-166. http://dx.doi.org/10.1590/S1983-14472011000100021

Sit, D. K. \& Wisner, K. L. (2009). The identification of postpartum depression. Clinical obstetrics and gynecology, 52(3), 456. http://dx.doi.org/10.1097/GRF.0b013e3181b5a57c

Autores:

BÁRBARA CAMILA DE CAMPOS - Mestranda, Universidade Estadual Paulista Júlio de Mesquita Filho.

Olga Maria Piazentin Rolim Rodrigues - Livre-docente em Psicologia pela Universidade Estadual Paulista Júlio de Mesquita Filho.

Endereço para correspondência:

Bárbara Camila de Campos

Universidade Estadual Paulista Júlio de Mesquita Filho

Faculdade de Ciências de Bauru, Departamento de Psicologia

Av. Luiz Edmundo Carrijo Coube, s/n - Vargem Limpa

CEP 17033-360 Bauru, SP, Brasil

E-mail: badecampos@gmail.com

Recebido em: 19.05 .15

Aceito em: 10.05 .15 